\section{A New Type of Focusing X-Ray Monochromator}

FollowiNg up a suggestion which arose during a conversation with Dr. T. Ll. Richards recently, I have been experimenting with a new type of focusing $\mathrm{X}$-ray monochromator. The material used is single texture copper strip, as described by Cook and Richards 1,2. This material has a very high degree of preferred orientation, with a $<100>$ direction lying in the rolling direction, and a $\{100\}$ plane in the strip surface. The orientation is suitable for curved monochromators set to reflect 200 , and since the copper can be bent and ground much more easily than the crystals usually used for this purpose, and is also much more robust, its advantages a re obvious. The reflected beam is quite intense and can easily be traced with a fluorescent screen.

The development of this type of monochromator is continuing, and fuller details will be published later.

$$
\begin{gathered}
\text { Metallurgy Department, } \\
\text { The University, }
\end{gathered}
$$$$
\text { May } 10 .
$$

${ }^{1}$ Cook and Richards, J. Inst. Metals, 66, 1 (1940).
${ }^{2}$ Cook and Richards, J. Inst. Metals, 67, 203 (1941).

W. H. HALI

\section{Two Relativistic Modeis of Dirac's Electron}

THE view has often been expressed that some at least of the difficulties encountered in the quantum-mechanical theory of the electron arise from the inadequateness of the underlying classical model. Perhaps some progress might be achieved by substituting a spin particle obeying the laws of relativistic dynamics for the spinless particle used as starting-point by Dirac. At least two different relativistic models of an electron (or, more generally, of any elementary particle with spin, as it seems probable that it is only through the process of quantization that the individuality of different sorts of particles is brought in) may be thus constructed, corresponding to the first and the second cases dealt with in earlier communications ${ }^{1}$. I shall only comment here on the second model.

This consists of a unipole-dipole singularity moving with the velocity of light according to the laws of relativistic dynamics. The essential point, however, is that it is not the singularity itself, but rather its mean position-or the small circle on which it moves- which has to be considered as representing the electron. The following is a list of some analogies and some promising differences between my second model of an electron and its quantum-mechanical counterpart. (a) G has ceased to be parallel to $\nabla$ just as in Dirac's theory, where the linear momentum and the velocity have different operators. $(b)$ The scalar momentum and the velocity have different operators. (b) The scalar
velocity of the singularity is always $c$, whereas the scalar velocity of velocity of the singularity is always $c$, whereas the scalar velocity of
the circle as a whole may acquire all values between 0 and $c$. (c) In the circle as a whole may acquire all values between 0 and $c$. $(c)$ In
both theories the moments of momentum are not constants of motion by themselves but they may be easily supplemented in such a way as to acquire that property ; the additional terms involve the vector to acquire that property ; the additional terms involve the vector a very similar manner. It might be objected that the above items are expressly brought in to stress the a nalogy, but in any event the followexpressly brought in to stress the analogy, but in any event the followof relativity and without any new arbitrary assumptions. $(d)$ If we put $M_{\sigma}$ equal to the mass of an electron and $\delta_{\sigma}$ equal to $\hbar / 2$, the frequency of revolution around the circle will be just equal to the frequency of Schrödinger's Zitterbewegung; and $(e)$ the proper diameter of the circle $2 r_{\sigma}=\hbar / M_{\sigma} c$ becomes equal to the maximum accuracy with which the position of a particle of mass $M \sigma$ can be ascertained. The amplitude of the Zitterbewegung has the same order of magnitude. (f) Two isotropic tensors, namely, $w^{\alpha}$, for which $w_{\alpha} w^{\alpha}=0$, and $s^{\alpha \beta}$ for which $s_{\alpha \beta} s^{\alpha \beta}=0$ play a dominant part in our theory ; it is well known that isotropic four-vectors and isotropic four-dimensional bivectors are closely connected to spinors.

$(g)$ The motion of the spin singularity in an electromagnetic fleld is far more complicated than in a fleld-free space, but as a first approximation, if the intensity of the field is small enough, we may go on speaking of motion in a circle even in an electromagnetic fleld, the circle being subject to a small acceleration as a whole and to slight deformations. Obviously the inequality to be fulflled by the intensity of the field may be obtained by expressing the fact that in the rest system of the eircle the displacement of the centre of the circle during the time of one revolution should be vanishingly small in comparison with the proper radius of the circle. The resulting inequality (in the case of an electrostatic fleld of intensity $E$ )

$$
\varepsilon E \hbar \ll M_{\sigma^{2} c^{3}},
$$

is just the same as the condition of no jumps from positive to negative energy states in Dirac's theory.

(h) Variability of mass and pair production. Strictly speaking, all that has been said hitherto lacks real foundation so long as we have not proved that $\boldsymbol{M}_{\boldsymbol{\sigma}}$ is a constant of motion, and that it may hence be put equal to the mass of an electron. Less restrictively, the question may be put as follows. At the outset, let the electron move in a fleldfree space ; it is then represented by a circle of constant proper radius $r_{\sigma}$, constant mass $M_{\sigma}$ and constant spin $8_{\sigma}$. Now, let the electron enter an electromagnetic field and, after remaining there for a while, get out of it into a fleld-free space again; there it becomes once more a regular eircle (the rest-system of which is in general different from what it was before passage through the field). But do $M_{\sigma}$, and hence $r_{\sigma}$ and $8_{\sigma}$, return to their previous values? Strictly speaking, the answer is in the negative, but it may be proved that $M_{\sigma}$ is approximately constant when the fleld fulflis a certain condition, which for the fleld of a plane monochromatic wave of frequency $v$ reduces to the inequality

$$
h \nu \ll 2 M_{\sigma^{\prime 2}} \text {. }
$$

Thus we see that the condition of constancy of mass in the classical theory is equivalent to the condition of non-production of pairs in quantum-mechanics. In other words, it may be expected that it is only through the process of quantization that the mass of the electron becomes constant-so long as it is not created or annihilated by pair production.

It is not at all clear how the quantization of our relativistic model has to be performed. In any event, it will have to be done in a very different manner from the present one, for Schrödinger's $Z$ itterbewegung is a consequence of the superposition of states of positive and negative energies : to-day we imagine that a particle is either in a state of positive or in a state of negative energy, whereas our circular model of an electron, being a model of the Zitterbewegung, must correspond simultaneously to states of positive and of negative energies.

The full details of the theories referred to in this and earlier communications will be published in five papers in the flrst post-war issue of Acta Physica Polonica.

Institute of Theoretical Physics

JAN W. WEYSSENHOFF

Jagellonian University,

$$
\begin{aligned}
& \text { Cracow. } \\
& \text { Jan. } 3 .
\end{aligned}
$$

${ }^{2}$ Nature, 157, 766, 809 (1946).

\section{Deflexion of Light in the Gravitational Field without using Einstein Geometry}

IN a communication in Nature ${ }^{1}$, Cheng has shown that the advance of perihelion can be easily obtained from a classical Lagrangian with contraction of length and time in the gravitational fleld but without the use of the Einstein geometry. Applying this same result to a beam of light passing through the sun's surface with $h=r^{2} \dot{\theta} \beta^{2} \doteq r_{0} c$, it is found that the deflexion angle would be $2 \cdot 61^{\prime \prime}$.

The equation of path is

$$
\frac{d^{2} \mu}{d \theta^{2}}+\mu=\frac{G M}{h^{2}}+3 \lambda \mu^{2} .
$$

The approximate solution to the flrst order of $G M / c^{2}$ is

$$
\mu=\frac{1}{r_{0}} \cos \theta+\frac{G M}{h^{2}}(5+\cos (\pi+2 \theta)) .
$$

Therefore, the angle of deflexion $\varepsilon$ is obtained from the equation

$$
\begin{gathered}
\mu=\left\{\cos \left(\frac{\pi}{2}+\frac{t}{2}\right)+\frac{G M r_{0}}{h^{2}}(5-\cos (\pi+\varepsilon))\right\} \frac{1}{r_{0}}=0 \\
\varepsilon=\frac{6 M G}{r_{0} c^{2}}=2 \cdot 61^{\prime \prime} .
\end{gathered}
$$

This result seems in better agreement with corrected astronomical data than Einstein's original value, which is only two thirds of the above value.

$$
\text { Department of Physics, }
$$

SU-ChING KIANe

National University of Chekiang,

Meitan, Kweichow, China.

${ }^{2}$ Cheng, Nature. 155, 574 (1945).

\section{Statistical Thermodynamics of Mixtures}

From some work employing the Bethe technique, I deduced ${ }^{1}$ formula for the number of configurations of mixtures of $p$-mer an single molecules on a lattice. Afterwards, a kinetic derivation of this formula, together with its generalization to the case of a mixture of simple or branched chain molecules of any number of species, was given by Guggenheim ${ }^{2}$ using the principle of deta
formula in question can be written in the form :

$$
\begin{gathered}
\log g\left(N_{i}\right)=\frac{1}{2} z \log \left(\sum_{i} q_{i} N_{i}\right) !-\sum_{i} \log N_{i} !- \\
\left(\frac{1}{2} z-1\right) \log \left(\sum_{i} r_{i} N_{i}\right) !+\sum_{i} N_{i} \log p_{i} .
\end{gathered}
$$

where $q_{i} z$ is the number of sites which are closest neighbours of a molecule of species $i$ which contains $r_{i}$ submolecules (a slight modiflcation of the definition covers the case of flexible open-chain molecule which can bend back on themselves), and the other symbols have
their customary meanings. The value of $q_{i}$ is given by

$$
z\left(r_{i}-q_{i}\right)=2\left(r_{i}-1\right) \quad(\text { all } i)
$$

In my work only simple chain molecules were considered. Guggen heim's derivation is equally applicable to molecules with branched chains as to simple-chain molecules, but "the exclusion of molecules having closed rings is an essential condition" for Guggenheim's argu- 